\title{
Coulisses
}

Revue de théâtre

6 | Printemps 1992

Varia

\section{Combat de nègre et de chiens}

\section{(2) OpenEdition}

1 Journals

Édition électronique

URL : http://journals.openedition.org/coulisses/1909

DOI : 10.4000/coulisses.1909

ISSN : 2546-9460

\section{Éditeur}

Presses universitaires de Franche-Comté

\section{Édition imprimée}

Date de publication : 1 juin 1992

ISSN : 1150-594X

\section{Référence électronique}

"Combat de nègre et de chiens », Coulisses [En ligne], 6 | Printemps 1992, mis en ligne le 15 mars

2019, consulté le 21 octobre 2019. URL : http://journals.openedition.org/coulisses/1909 ; DOI :

10.4000/coulisses.1909

Ce document a été généré automatiquement le 21 octobre 2019.

Coulisses 


\section{Combat de nègre et de chiens}

Combat de nègre et de chiens de Bernard-Marie Koltès est une création du Centre Dramatique National de Franche-Comté. La pièce a été jouée du 7 au 18 avril et du 5 au 9 mai 1992 au petit théâtre du CDN.

1 Ecrit en 1979, Combat de nègre et de chiens a connu quatre éditions : tapuscrit (c'est sous cette forme que Chéreau a lu la pièce de Koltès), Théâtre-Ouvert-Stock, Collection des Amandiers, éditions de Minuit. En France, la pièce a été créée en 1983 dans une mise en scène de Patrice Chéreau avec Michel Piccoli, Philippe Léotard et Myriam Boyer.

2 Curieusement, les pièces de Koltès ont souvent été créées d'abord à 1'étranger, en traduction avant d'être représentées en France ${ }^{1}$. Ce fut le cas pour Combat de nègre et de chiens, dont la première mondiale eut lieu sur une scène américaine, dans une traduction de Matthew Ward. C'est en effet au théâtre de La Mamma à New-York que la pièce fut initialement jouée, dans une mise en scène de Françoise Kourilsky. Même détour pour Quai Ouest, dont la première représentation se déroula à Amsterdam (1985), et pour Roberto Zucco créé à Berlin (1990). Il faut d'ailleurs se rendre à l'évidence : Koltès est plus fréquemment joué à l'étranger qu'en France. Est-ce le fait d'une certaine tradition théâtrale française qui a peu de goût pour les productions simultanées ? Doiton y lire «l'effet Chéreau », metteur en scène "officiel » des textes de Koltès, dont la stature paralyserait la création dramatique peu encline à se mesurer à lui ? La situation est très différente en Allemagne où Combat de nègre et de chiens a été mis en scène 17 fois au cours des huit dernières saisons théâtrales, dont 5 en 90-91. Plus généralement, les pièces de Koltès sont régulièrement représentées dans les théâtres allemands : en huit ans, on dénombre 58 productions; pour la seule saison 90-91, on compte 26 mises en scène de textes de Koltès dont 10 de sa dernière pièce Roberto Zucco.

3 Il n'est donc pas étonnant, dans de telles conditions, que Koltès soit mal connu du public bisontin qui, avec les représentations données au CDN, a découvert un auteur. 


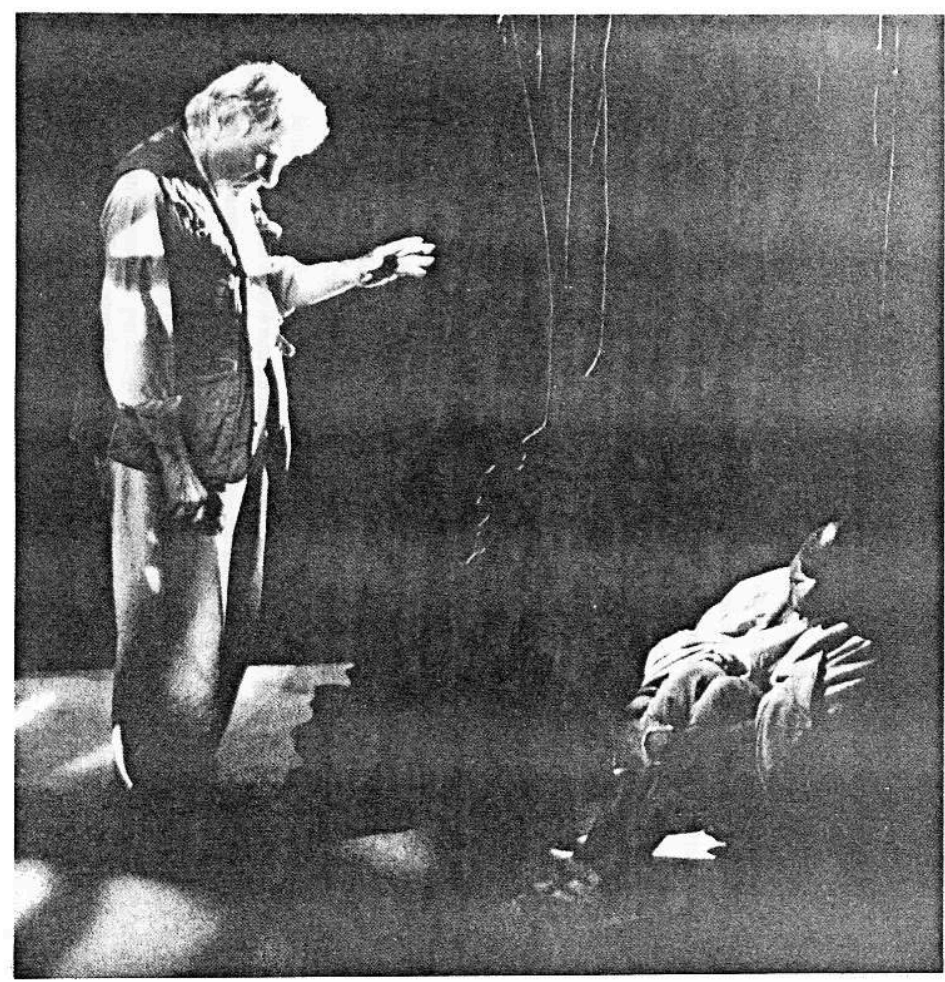

Photo Lot

\section{René LOYON parle de Combat de nègre et de chiens}

Extrait d'une interview menée par Lucien Attoun, diffusée en direct sur France-Culture, vendredi 24 avril 1992.

\section{L.A. : Pourquoi Koltès?}

R.L. : C'était un vieux projet puisque j'avais lu le texte en 1980 dans l'édition du tapuscrit et, dès cette première lecture qui était pour moi une découverte de Koltès, j'ai eu envie de monter ce texte. De plus, il s'inscrivait dans une histoire un peu plus personnelle: je venais de monter Les voyages avant l'an 40, adaptation du journal intime de mon propre grand-père, magistrat colonial dont l'essentiel de la carrière s'est déroulé en Afrique. Il y avait pour moi un lien très évident entre cet homme, Français, bourgeois, catholique lyonnais qui tenait un journal dans lequel il y avait toutes sortes de considérations sur l'Afrique et le texte de Koltès qui nous donne de l'Afrique une vision assez sombre.

\section{L.A. : Est-ce une vraie Afrique ? On dit que c'est l'Afrique de Barbès ou de Pigalle?}

R.L. : Koltès s'est toujours défendu à propos de ce texte d'avoir voulu représenter l'Afrique et les Africains. Il est vrai qu'il évoque plutôt une Afrique un peu rêvée, fantasmatique. Mais en même temps, c'est aussi le souvenir du voyage qu'il a fait au Nigeria et, dans la pièce, il y a un personnage d'Africain tout à fait en chair et en os, même s'il a une dimension symbolique ou mythique qui est très importante. Quand on met en scène un texte comme celui-ci, on navigue constamment entre la 
dimension concrète, réaliste de l'Afrique et un rêve d'Afrique tel que Koltès a pu en parler.

L.A. : Dans ce texte, c'est l'Afrique qui n'a pas encore rompu avec son passé; la colonisation est encore présente avec les gens paternalistes et durs qui font des affaires. Et finalement l'avenir n'appartient ni à l'un ni à l'autre.

R.L. : Malheureusement, la pièce a pris en dix ans, une actualité de plus en plus aiguë. Il y a dix ans, elle paraissait curieuse. Je me souviens que l'une des premières impressions que j'avais retirées de cette première lecture, c'était celle d'une étrangeté du style. Ce qui me frappe maintenant, c'est que 1'actualité a d'une certaine façon rejoint l'écriture de Koltès.

L.A. : Vous avez souligné cette phrase de Koltès qui disait : « Ma patrie, c'est la langue »...

R.L. : C'est presque banal de le dire, mais une des raisons essentielles qui font, au-delà des questions thématiques, que l'on s'intéresse à un auteur, c'est sa qualité poétique. Or, quand on ouvre un livre de Koltès, on est dès la première ligne frappé par la densité poétique de son écriture.

L.A. : Lorsqu'on écoute bien l'écriture de Koltès, on s'aperçoit qu'il y a une difficulté à mettre en scène car il y a des monologues ou des récits, qui ne sont pas en réalité du roman transposé, mais qui sont du théâtre.

R.L. : Tous les acteurs qui ont eu à affronter un texte de Koltès ont dit cette difficulté. Et moi, malgré une familiarité assez ancienne avec le texte de Koltès, j'ai découvert le problème. On a souvent le sentiment de monologues entrecoupés par quelques phases d'action. Combat de nègre et de chiens est une pièce autour de conversations, les mots sont l'action, aussi les acteurs ont souvent l'impression de rester un peu en place à dire du texte, à échanger des répliques et on a beaucoup de mal à saisir très exactement ce qui s'y passe.

\section{L.A. : Comment a réagi le public de Besançon?}

R.L. : Il y a eu une certaine surprise, comme une circonspection, dans la façon dont le public a réagi. Mais ça se passe très bien. Quand je parle de circonspection, je veux dire que, contrairement à ce que nous pourrions imaginer, nous, gens de théâtre qui savons à quel point Koltès est un grand auteur, le public ne sait pas du tout qui est Koltès. Les spectacles mis en scène par Chéreau ont eu un grand succès à Paris mais ils ont peu tourné. Il y a eu très peu d'échos donnés à cette œuvre. On s'aperçoit avec émotion que le public bisontin découvre Koltès et que les gens sont très touchés par cet auteur.

\section{Le choix du petit théâtre (extrait d'un entretien)}

J'ai choisi de présenter Combat de nègre et de chiens dans le petit théâtre parce que, pour moi, c'est un théâtre qui relève plus de la musique de chambre que de la grande musique symphonique. J'ai envie d'entendre ces quatre instruments que sont les personnages travailler dans le murmure et le chuchotis. C'est une pièce où l'on converse énormément (...). Il faut se permettre le luxe de l'écouter tranquillement, dans un rapport intime aux acteurs.

\section{Impressions du public :}

C'est pour moi un bon spectacle et une bonne occasion de découvrir un auteur. J'ai aimé le jeu des comédiens, mais je n'ai pas apprécié la fin, avec le décalage entre le 
jeu réaliste tout au long du spectacle et l'allégorie de la négritude derrière le fleuve. De façon générale, le décor m'a paru surutilisé.

J'ai trouvé le décor prétentieux, avec l'utilisation de procédés cinématographiques sans en avoir les moyens. J'ai beaucoup aimé l'interprétation d'Alboury, dense, sobre.

J'ai découvert un texte que je ne connaissais pas et ce n'est déjà pas si mal. J'ai été sensible au travail des comédiens, sauf peut-être le personnage féminin qui m'a paru peu affirmé, moins existant que les autres, mais, connaissant mal la pièce, je suis incapable de dire si cela vient du personnage ou de l'interprétation.

\section{Leone, une idée des vies successives.}

Ce que je crois, moi, c'est qu'à la première vie, on doit être un homme comme ce Cal, l'horrible type ; ces hommes-là comprennent si peu de choses, ils sont si bêtes, oh, si bouchés, il faut bien qu'ils en soient à leur toute première vie, les bandits ! Je crois que c'est seulement après beaucoup de vies d'homme, ridicules et bornées, brutales et braillardes comme sont les vies des hommes, que peut naître une femme. Et seulement, oui seulement après beaucoup de vies de femme, beaucoup d'aventures inutiles, beaucoup de rêves irréalisés, beaucoup de petites morts, alors seulement, alors peut naître un nègre, dans le sang duquel coulent plus de vies, et plus de morts, plus de brutalités et d'échecs, plus de larmes que dans aucun autre sang. Et moi, combien de fois devrai-je mourir encore, combien de souvenirs et d'expériences inutiles devront encore s'entasser en moi ?

Il y a bien une vie que je finirai par vivre pour de bon, non?

Extrait des Carnets de Combat de nègre et de chiens, éd. de Minuit, pp. 124-125.

\section{NOTES}

1. Sur la diffusion des pièces de Koltès à l'étranger, et particulièrement en Europe, voir Théâtre/ Public n 105, mai- juin 1992, pp.59-68. 